\title{
Renal histopathological findings in relation to ambulatory blood pressure in chronic kidney disease patients
}

\author{
Kotaro Haruhara, Nobuo Tsuboi, Kentaro Koike, Akira Fukui, Yoichi Miyazaki, Tetsuya Kawamura, \\ Makoto Ogura and Takashi Yokoo
}

Recent studies have demonstrated that ambulatory blood pressure monitoring is useful for predicting the long-term renal prognosis and future cardiovascular events in chronic kidney disease patients. Currently, however, information is limited regarding the relationships between individual renal histopathological findings and abnormalities in ambulatory blood pressure. This retrospective cross-sectional study included a total of 138 patients, in whom both renal biopsies and ambulatory blood pressure monitoring were performed during the same admission period. Renal histopathological findings, including global glomerulosclerosis, interstitial fibrosis/tubular atrophy and the presence of arterial lesions and arteriole lesions, were scored and analyzed in relation to the ambulatory blood pressure values. Among these histopathological characteristics, only the severity of interstitial fibrosis/tubular atrophy exhibited a significant association with an increased mean value of daytime and nighttime blood pressure. However, the remaining histopathological features showed only trends or weak relationships with these values. In addition, a moderately advanced grade of interstitial fibrosis/tubular atrophy was found to be significantly associated with both daytime and nighttime hypertension, independent of the kidney function, overt proteinuria and the use of antihypertensive medications, according to multivariate analyses. Furthermore, the night-to-day ratio of the mean blood pressure displayed a significant increasing trend according to the grade of interstitial fibrosis/tubular atrophy. These results suggest that interstitial fibrosis/tubular atrophy is the most relevant renal histopathological parameter associated with abnormalities in ambulatory blood pressure, including nocturnal hypertension, in this population.

Hypertension Research (2015) 38, 116-122; doi:10.1038/hr.2014.140; published online 18 September 2014

Keywords: ambulatory blood pressure monitoring; chronic kidney disease; interstitial fibrosis/tubular atrophy; nocturnal hypertension; renal biopsy

\section{INTRODUCTION}

Hypertension is a principal cause of chronic kidney disease (CKD) and an exacerbating factor of preexisting CKD. In fact, hypertension is found in at least $70 \%$ of patients with CKD. ${ }^{1}$ In particular, nocturnal hypertension is often observed with ambulatory blood pressure monitoring (ABPM) in CKD patients. ${ }^{2,3}$ In addition, it has been reported that the detection of nocturnal hypertension in patients with ABPM is a useful finding for predicting a poor renal prognosis and future cardiovascular events in such patients. ${ }^{4,5}$ Therefore, ABPM may be more useful than office or home blood pressure (BP) measurement in the management of BP abnormalities in CKD patients. In another cross-sectional study, the s.d. of the daytime BP in the ABPM was strongly correlated with the renal vascular resistance assessed by renal Doppler ultrasonography in hypertensive patients. Thus, ABPM is a more efficacious index for evaluating very early renal damage than the estimated glomerular filtration rate (eGFR). ${ }^{6,7}$
Hypertension in the setting of CKD is caused by various factors, including salt sensitivity and increased activity of the renin-angiotensin and sympathetic nervous systems. ${ }^{8,9}$ Importantly, most factors related to the pathogenesis of hypertension are also associated with renal tissue injury, suggesting that the factors contributing to both BP abnormalities and renal tissue injury share similar pathophysiological mechanisms. Currently, however, little is known regarding the relationships between renal histopathological findings and BP abnormalities, including those analyzed using ABPM in CKD patients. In the current study, therefore, we aimed to determine the relationships between individual renal histopathological parameters and ABPM findings in CKD patients treated with renal biopsies. Investigating the renal histopathological findings associated with abnormalities in the ABPM may help identify the lesions responsible for BP regulation within the injured kidneys in CKD patients. 


\section{METHODS}

\section{Settings and participants}

This retrospective cross-sectional study included consecutive patients who underwent percutaneous native renal biopsies and ABPM at the Jikei University Hospital, Tokyo, Japan, during the period from April 2010 to March 2013. Patients with acute kidney injury, nephrotic syndrome and rapidly progressive glomerulonephritis were excluded from the analysis. Both the renal biopsies and ABPM were performed during the same hospitalization period. The patients' sodium intake (6g per day) was restricted during hospitalization. Diabetes mellitus was defined as a value of HbAlc $>6.5 \%$ (National Glycohemoglobin Standardization Program (NGSP)) or as patients under medical treatment for diabetes mellitus at the time of renal biopsy. The eGFR was calculated by applying a modified three-variable equation for estimating the GFR for Japanese patients: ${ }^{10} \mathrm{eGFR}=194 \times \mathrm{Age}^{-0.287} \times \mathrm{s}-\mathrm{Cr}^{-1.094}(\times 0.739$, if female), where s-Cr is the serum creatinine. All patients included in this study provided their written informed consent for the renal biopsy. The study protocol was approved by the ethics review board of the Jikei University School of Medicine (24-317 7083).

\section{ABPM analysis}

ABPM was performed when possible in all CKD patients with or without hypertension 4-7 days after admission and 2-5 days after the renal biopsy to evaluate the circadian $\mathrm{BP}$ and the possible risk of future progression to renal failure. The ABPM analyses were performed using a TM-2431C (A\&D, Tokyo, Japan) device. The bladder size of the device was $22 \mathrm{~cm} \times 12 \mathrm{~cm}$. The BP was measured every $30 \mathrm{~min}$ during the day and every $60 \mathrm{~min}$ at night. We defined the BP parameters according to the Japanese Society of Hypertension guidelines as follows: (i) daytime and nighttime BP were defined as the mean value of the daytime and nighttime BP, respectively; (ii) daytime hypertension was defined as a daytime BP of $>135 \mathrm{~mm} \mathrm{Hg}$ for systolic BP and $>85 \mathrm{~mm} \mathrm{Hg}$ for diastolic $\mathrm{BP}$, and nighttime hypertension was defined as a nighttime $\mathrm{BP}$ of $>120$ $\mathrm{mm} \mathrm{Hg}$ for systolic BP and $>70 \mathrm{~mm} \mathrm{Hg}$ for diastolic BP; (iii) the night-to-day ratio (NDR) was defined as the ratio of the nighttime mean $\mathrm{BP}$ to the daytime mean BP, ${ }^{11}$ and NDR of 0.9 was used as a threshold to distinguish a nondipper and dipper pattern of the circadian rhythm of BP. ${ }^{12}$ The change in the nocturnal BP was calculated as $100 \times(1-\mathrm{NDR})$. A dipping status regarding the $\mathrm{BP}$ was defined as follows: extreme dipper $=$ the decrease in nocturnal BP was $\geqslant 20 \%$; dipper $=$ a decrease in nocturnal BP of $\geqslant 10 \%$ and $<20 \%$; nondipper $=$ a decrease in nocturnal $\mathrm{BP}$ of $>0$ and $<10 \%$; and reverse dipper $=$ a decrease in nocturnal $\mathrm{BP}$ of $<0 \%$. $^{12}$

\section{Histopathological analysis}

All renal tissue specimens were obtained via percutaneous needle biopsies. The tissues were embedded in paraffin, cut into 3- to $4-\mu \mathrm{m}$ sections and stained with hematoxylin-eosin, periodic acid-Schiff, Masson's trichrome and periodic acid-methenamine silver stain. Biopsies containing $<5$ glomeruli were regarded to be inadequate for scoring and were excluded from the analysis. Four candidate chronic renal histopathological findings, including global glomerulosclerosis (GS), interstitial fibrosis/tubular atrophy (IF/TA) and the presence of arterial lesions ( $\mathrm{AA}$ ) and arteriole lesions (AO), were evaluated. The histopathological findings were independently evaluated by two well-trained nephrologists (KH and NT). The patients' information was blinded at the time of the histopathological evaluation, and the identified values were averaged before grading. Representative photomicrographs of each histological grade are presented in Figure 1. The histopathological grading of the four parameters was performed according to the definitions of the Oxford classification, as originally proposed for the evaluation of IgA nephropathy. ${ }^{13}$ The grade of GS was defined based on the ratio of the number of sites of GS to the total number of glomeruli identified on the biopsy specimen, as follows: grade $0=<5 \%$, grade $1=5-25 \%$ and grade $2=>25 \%$. The grade of IF/TA was evaluated according to semiquantitative methods. We defined a severity of IF/TA of $<5 \%$ as grade $0,5-25 \%$ as grade 1 and $>25 \%$ as grade 2 . The grade of AA was scored according to the most severe lesions, and intimal thickening was scored by comparing the thickness of the intima to that of the media in the same segment of the vessel. We defined a severity of AA involving the absence of intimal thickening as grade $0,50 \%$ or less as grade 1 and $>50 \%$ as grade 2 . The grade of $\mathrm{AO}$ was evaluated based on the proportion of arteriolar arteries exhibiting hyalinosis as follows: grade $0=a$ proportion of lesions of $<5 \%$, grade $1=$ a proportion of lesions of $5-25 \%$ and grade $2=$ a proportion of lesions of $>25 \%$.

\section{Statistical analysis}

A one-way analysis of variance was used to analyze the trends in the mean BP values in relation to each histopathological finding. A Kruskal-Wallis analysis was used to compare the prevalence of daytime and nighttime hypertension, and a frequency of NDR of $>0.9$ for each histopathological grade. Univariate and multivariate logistic analyses were applied to identify the factors associated with daytime and nighttime hypertension. The relevant parameters or the variables that were significantly associated on the basis of a univariate analysis were included in the multivariate analysis. The data were analyzed using a commercially available software program (SPSS version 16.0, Chicago, IL, USA). Values of $P<0.05$ were considered to be significant in all statistical tests.

\section{RESULTS}

Clinical characteristics of the subjects at the time of the biopsy

We initially enrolled 256 consecutive patients who underwent a native renal biopsy. Patients were excluded if they had acute kidney injury or rapidly progressive glomerulonephritis $(n=36)$, nephrotic syndrome $(n=50)$ or an inadequate renal biopsy specimen of fewer than five glomeruli $(n=2)$. Among the remaining 168 patients, 138 patients (82\%) received ABPM. The clinicopathological characteristics of the 30 patients who did not receive ABPM were similar to the 138 patients included in this study, except that the 30 patients without ABPM had lower eGFRs at the time of biopsy. The clinical characteristics of the subjects at the time of the biopsy are summarized in Table 1. Approximately half of the patients had a GFR category of 1 or 2, and the other half had a GFR category of $3 a, 3 b, 4$ or 5 . The mean amount of urinary protein excretion of the patients was $1.24 \mathrm{~g}$ per day. Almost half of the patients received antihypertensive medications. Thirty-nine percent of the patients in the study received angiotensinconverting enzyme inhibitors or angiotensin type- 1 receptor blockers, $11 \%$ of whom also received diuretics. The distribution of the histological diagnosis was as follows: $73(53 \%)$ patients were diagnosed with IgA nephropathy, 15 (11\%) were diagnosed with benign nephrosclerosis, 12 (9\%) were diagnosed with idiopathic membranous nephropathy, nine $(6 \%)$ were diagnosed with focal segmental glomerulosclerosis, three $(2 \%)$ were diagnosed with diabetic glomerulosclerosis and 26 (19\%) were diagnosed with other conditions. The IF/ TA grade exhibited significant correlations with the GS, AA and AO grade (data not shown).

\section{Relationships between the histopathological parameters and the mean ambulatory BP}

The relationships between the mean ambulatory $\mathrm{BP}$ and the histopathological parameters were analyzed using a one-way analysis of variance (Table 2). The grade of IF/TA was associated with the mean values of the daytime and nighttime systolic and diastolic BP on ABPM (each $P<0.001$ ). The grade of $\mathrm{AO}$ was associated with the mean values of the daytime systolic $(P=0.003)$, the diastolic BP $(P=0.011)$ and the nighttime diastolic $\mathrm{BP}(P=0.034)$, but not the nighttime systolic BP. In contrast, the grades of GS and AA did not show any statistically significant relationships with the BP values. The significant relationships between the IF/TA grade and the mean values of daytime and nighttime BP remained in the analyses of the patients without apparent renal impairment (eGFR $60 \mathrm{ml} \mathrm{min}^{-1}$ per $1.73 \mathrm{~m}^{2}$ or more), those treated without antihypertensive medications, female 


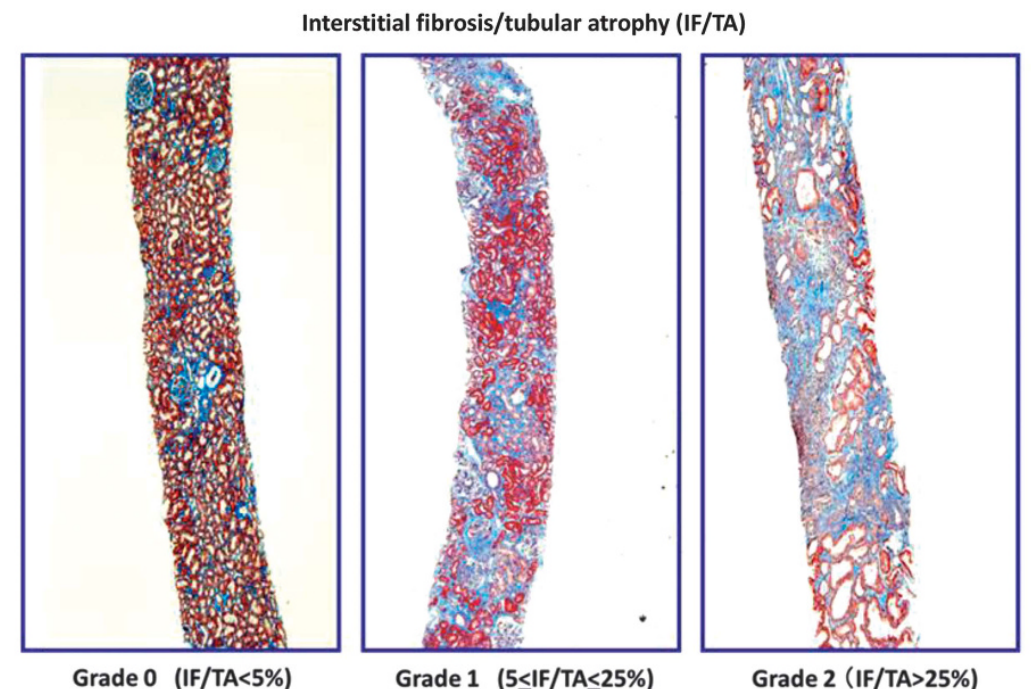

b

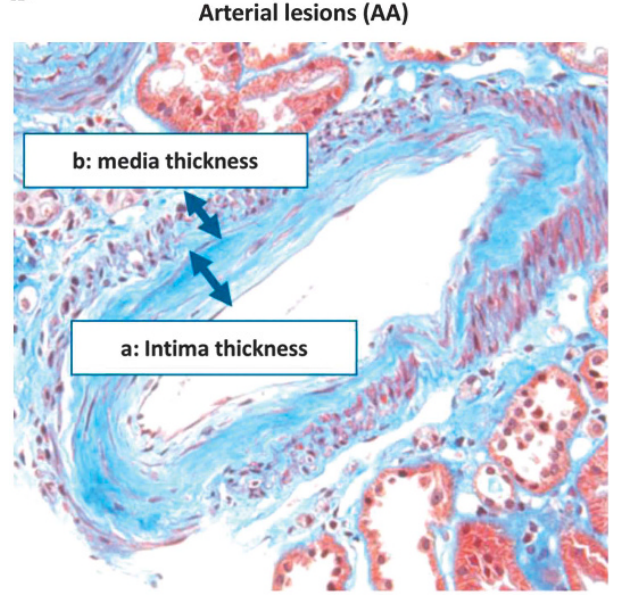

C
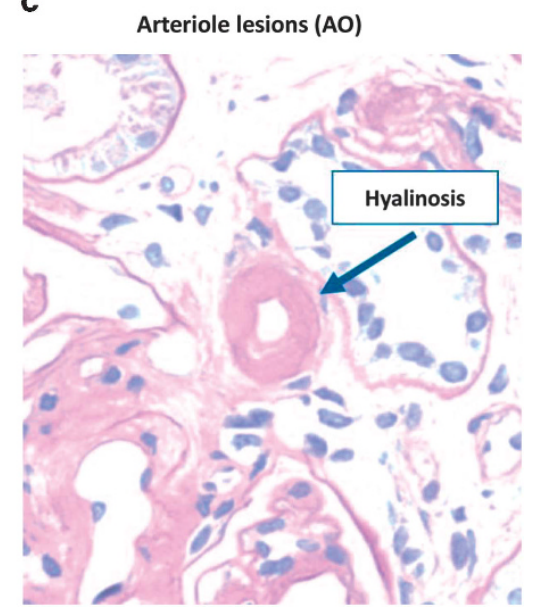

Figure 1 Representative photomicrographs of each histological grade. (a) The grade of interstitial fibrosis/tubular atrophy (IF/TA) was evaluated according to semiquantitative methods, as follows: grade $0=<5 \%$, grade $1=5-25 \%$ and grade $2=>25 \%$ (Masson-trichrome stain, original magnification $\times 50$ ). (b) The grade of arterial lesions ( $A A$ ) was defined based on the most severe lesions of intimal thickening by comparing the thickness of the intima (a) to that of the media (b) in the same segment of the vessel. The grade of AA was determined based on the proportion of these values (a/a+b), as follows: grade $0=a b s e n c e$ of intimal thickening, grade $1=$ intimal thickening of $50 \%$ or less and grade $2=$ intimal thickening of $>50 \%$ (Masson-trichrome stain, original magnification $\times 200$ ). (c) The grade of arteriole lesions (AO) was defined based on the proportion of sites of arteriolar hyalinosis, as follows: grade $0=<5 \%$, grade $1=5-25 \%$ and grade $2=>25 \%$ (periodic acid-Schiff stain, original magnification $\times 400$ ).

patients and nondiabetic patients (data not shown). In contrast, the grade of AO did not exhibit any statistically significant associations with the daytime or nighttime BP values in the analyses of these subgroups (data not shown).

Circadian abnormalities in BP were also analyzed. Of the patients included in this study, $6(4 \%)$ were extreme dippers, $52(38 \%)$ were dippers, $63(46 \%)$ were nondippers and $17(12 \%)$ were reverse dippers. Figure 2 shows the frequency of an NDR of $>0.9$ for each IF/TA grade. The frequency of an NDR $>0.9$ increased in association with the IF/TA grade, exhibiting a statistically significant trend $(P=0.032)$. This trend remained in the analyses of the patients without apparent renal impairment $(n=70, P=0.007)$ and those treated without antihypertensive medications $(n=71, P=0.023)$. In contrast, there were no significant relationships between an NDR $>0.9$ and the grades of the other histopathological findings, including GS, $\mathrm{AA}$ and $\mathrm{AO}$.
Relationships between the histopathological parameters and daytime and nighttime hypertension

Forty-nine $(36 \%)$ patients demonstrated daytime hypertension and $86(62 \%)$ patients displayed nighttime hypertension, as measured on ABPM. Among the 49 patients with daytime hypertension, 48 (98\%) patients showed nighttime hypertension.

To determine the effects of the renal function and the severity of IF/ TA on the abnormalities in the circadian BP, we examined the prevalence of nighttime hypertension in patients with or without IF/ TA grade $2(>25 \%)$ according to the GFR categories (Supplementary Figure). The prevalence of nighttime hypertension in patients with IF/ TA grade 2 tended to be higher than that in patients without IF/TA grade 2 in each GFR category. In particular, the prevalence of nighttime hypertension is clearly higher in the patients with IF/TA grade 2 , even in the patients with preserved renal function (GFR categories 1 and 2). 
Table 3 shows the results of the logistic regression analyses of the factors found to be associated with daytime and nighttime hypertension. In the multivariate analyses, both male gender and an IF/TA grade $2(>25 \%)$ exhibited significant associations with daytime and nighttime hypertension independent of other factors such as the

Table 1 Clinical characteristics at the time of the biopsy

\begin{tabular}{|c|c|}
\hline Variable & $N=138$ \\
\hline Age (year) & $45 \pm 15(15-83)$ \\
\hline Male $(n(\%))$ & $93(67)$ \\
\hline $\mathrm{BMI}\left(\mathrm{kg} \mathrm{m}^{-2}\right)$ & $23.1 \pm 3.45(16.6-37.0)$ \\
\hline Current smoker $(n(\%))$ & $33(24)$ \\
\hline Diabetes mellitus ( $n(\%))$ & $13(9)$ \\
\hline Urinary protein excretion (g per day) & $1.24 \pm 1.33(0.03-7.77)$ \\
\hline eGFR $\left(\mathrm{ml} \mathrm{min}{ }^{-1}\right.$ per $\left.1.73 \mathrm{~m}^{2}\right)$ & $60 \pm 28(5-134)$ \\
\hline \multicolumn{2}{|l|}{ GFR categories ( $n(\%))$} \\
\hline 1 & $19(14)$ \\
\hline 2 & $51(37)$ \\
\hline $3 a$ & $28(20)$ \\
\hline $3 b$ & $18(13)$ \\
\hline 4 and 5 & $22(16)$ \\
\hline \multicolumn{2}{|c|}{ Number of patients with hypertensive drugs (n (\%)) } \\
\hline 0 & $71(52)$ \\
\hline 1 & $21(15)$ \\
\hline 2 & $25(18)$ \\
\hline $3<$ & $21(15)$ \\
\hline Patients receiving RAS blockades ( $n(\%))$ & $54(39)$ \\
\hline Patients receiving diuretics ( $n(\%))$ & $15(11)$ \\
\hline
\end{tabular}

kidney function, overt proteinuria and the use of antihypertensive medications. We did not find any statistically significant factors associated with an NDR $>0.9$ in the multivariate analyses (data not shown).

\section{DISCUSSION}

The current results demonstrated that the severity of IF/TA based on the results of renal biopsy specimens exhibits significant associations with increased mean values of the daytime and nighttime BP in CKD patients who have undergone renal biopsies. However, other parameters, including GS, $\mathrm{AA}$ and $\mathrm{AO}$, showed only trends or weak relationships with the mean $\mathrm{BP}$ values. Moreover, the presence of moderately advanced IF/TA $(>25 \%)$ showed significant associations with both daytime $(>135 / 85 \mathrm{~mm} \mathrm{Hg})$ and nighttime hypertension ( $>120 / 70 \mathrm{~mm} \mathrm{Hg}$ ), which is independent of the kidney function, the

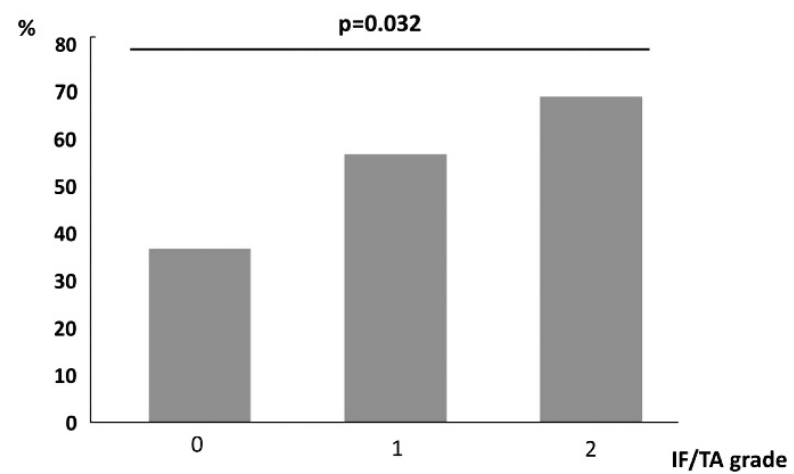

Figure 2 Frequency of an NDR $>0.9$ according to each interstitial fibrosis/ tubular atrophy (IF/TA) grade. The frequency of an NDR $>0.9$ increased in parallel with the grade of IF/TA; this trend was statistically significant $(P=0.032)$ by using a Kruskal-Wallis analysis. NDR, night-to-day ratio.

Table 2 Ambulatory BP and histological parameters: $N=138$

\begin{tabular}{|c|c|c|c|c|c|c|c|c|}
\hline \multirow{2}{*}{$\begin{array}{l}\text { Parameters } \\
\text { Grade (N of cases) }\end{array}$} & \multicolumn{2}{|c|}{ Daytime } & \multicolumn{2}{|c|}{ Nighttime } & \multicolumn{2}{|c|}{ Daytime } & \multicolumn{2}{|c|}{ Nighttime } \\
\hline & $S B P$ & $\mathrm{P}$-value & $S B P$ & P-value & $D B P$ & $\mathrm{P}$-value & $D B P$ & $\mathrm{P}$-value \\
\hline \multicolumn{9}{|l|}{ GS } \\
\hline $0(N=32)$ & $122 \pm 13$ & & $112 \pm 14$ & & $78 \pm 8$ & & $70 \pm 10$ & \\
\hline $1(N=57)$ & $123 \pm 16$ & & $114 \pm 18$ & & $79 \pm 10$ & & $73 \pm 11$ & \\
\hline $2(N=49)$ & $129 \pm 16$ & 0.071 & $121 \pm 18$ & 0.082 & $82 \pm 9$ & 0.191 & $74 \pm 10$ & 0.117 \\
\hline \multicolumn{9}{|l|}{ IF/TA } \\
\hline $0(N=21)$ & $120 \pm 11$ & & $111 \pm 12$ & & $77 \pm 7$ & & $68 \pm 9$ & \\
\hline $1(N=61)$ & $119 \pm 10$ & & $110 \pm 13$ & & $77 \pm 8$ & & $70 \pm 10$ & \\
\hline $2(N=56)$ & $134 \pm 19$ & $<0.001$ & $126 \pm 20$ & $<0.001$ & $84 \pm 10$ & $<0.001$ & $77 \pm 10$ & $<0.001$ \\
\hline \multicolumn{9}{|l|}{$A A$} \\
\hline $0(N=31)$ & $122 \pm 17$ & & $113 \pm 19$ & & $78 \pm 11$ & & $69 \pm 12$ & \\
\hline $1(N=68)$ & $125 \pm 15$ & & $117 \pm 16$ & & $80 \pm 9$ & & $74 \pm 10$ & \\
\hline $2(N=38)$ & $128 \pm 16$ & 0.217 & $119 \pm 19$ & 0.375 & $81 \pm 10$ & 0.475 & $74 \pm 10$ & 0.102 \\
\hline \multicolumn{9}{|l|}{$A O$} \\
\hline $0(N=38)$ & $120 \pm 15$ & & $114 \pm 17$ & & $77 \pm 9$ & & $70 \pm 11$ & \\
\hline $1(N=43)$ & $123 \pm 15$ & & $114 \pm 17$ & & $79 \pm 9$ & & $71 \pm 10$ & \\
\hline $2(N=56)$ & $131 \pm 16$ & 0.003 & $121 \pm 18$ & 0.066 & $83 \pm 9$ & 0.011 & $76 \pm 10$ & 0.034 \\
\hline
\end{tabular}

Abbreviations: AA, arterial lesions; AO, arteriole lesions; BP, blood pressure; DBP, diastolic blood pressure; GS, global glomerulosclerosis; IF/TA, interstitial fibrosis/tubular atrophy; SBP, systolic

The analyses were performed using a one-way analysis of variance. Each value of blood pressure is shown by mean \pm s.d. 
Table 3 Logistic analyses of daytime and nighttime hypertension: $N=138$

Daytime hypertension

\begin{tabular}{|c|c|c|c|c|c|c|c|c|}
\hline \multirow[b]{2}{*}{ Variables } & \multicolumn{2}{|c|}{ Univariate } & \multicolumn{2}{|c|}{ Multivariate } & \multicolumn{2}{|c|}{ Univariate } & \multicolumn{2}{|c|}{ Multivariate } \\
\hline & OR $(95 \% \mathrm{Cl})$ & P-value & OR $(95 \% \mathrm{Cl})$ & $\mathrm{P}$-value & OR $(95 \% \mathrm{Cl})$ & P-value & OR $(95 \% \mathrm{Cl})$ & P-value \\
\hline Age & $1.03(1.00-1.05)$ & 0.025 & $1.01(0.97-1.04)$ & 0.695 & 1.05 (1.02-1.08) & $<0.001$ & $1.03(0.99-1.07)$ & 0.142 \\
\hline Gender; male (vs. female) & $4.47(1.81-11.0)$ & 0.001 & $6.74(2.22-20.5)$ & 0.001 & $4.74(2.21-10.1)$ & $<0.001$ & $6.02(2.10-17.3)$ & 0.001 \\
\hline BMI & $1.04(0.94-1.15)$ & 0.415 & - & - & $1.18(1.05-1.33)$ & 0.006 & $1.12(0.98-1.29)$ & 0.091 \\
\hline Anti-hypertensive drugs; yes & $2.22(1.09-4.53)$ & 0.028 & $0.76(0.30-1.90)$ & 0.554 & $3.28(1.58-6.80)$ & 0.001 & $0.74(0.27-1.99)$ & 0.738 \\
\hline eGFR & $0.97(0.96-0.99)$ & $<0.001$ & $0.98(0.96-1.01)$ & 0.128 & 0.97 (0.95-0.98) & $<0.001$ & $0.99(0.96-1.02)$ & 0.412 \\
\hline UPE & $1.00(1.00-1.00)$ & 0.020 & $1.00(1.00-1.00)$ & 0.445 & $1.00(1.00-1.00)$ & 0.006 & $1.00(1.00-1.00)$ & 0.299 \\
\hline GS grade $2(v s$. grade $0+1)$ & $2.14(1.04-4.42)$ & 0.039 & $0.76(0.26-2.22)$ & 0.612 & $2.52(1.16-5.46)$ & 0.019 & $0.91(0.27-3.03)$ & 0.876 \\
\hline IF/TA grade 2 (vs. grade $0+1$ ) & $5.10(2.40-10.8)$ & $<0.001$ & $4.01(1.34-12.1)$ & 0.013 & $5.76(2.50-13.3)$ & $<0.001$ & $5.31(1.25-22.5)$ & 0.024 \\
\hline AA grade2 (vs. grade $0+1)$ & $2.07(0.96-4.46)$ & 0.063 & - & - & $2.06(0.90-4.71)$ & 0.085 & - & - \\
\hline AO grade 2 (vs. grade $0+1$ ) & $2.50(1.22-5.12)$ & 0.012 & $1.12(0.45-2.76)$ & 0.809 & $3.41(1.57-7.38)$ & 0.002 & $1.43(0.55-3.75)$ & 0.467 \\
\hline
\end{tabular}

Abbreviations: AA, artery lesions; AO, arteriole lesions; BMI, body mass index; CI, confidence interval; eGFR, estimated glomerular filtration rate; GS, global glomerulosclerosis; IF/TA, interstitial fibrosis/tubular atrophy; OR, odds ratio; UPE, urinary protein excretion.

overt proteinuria and the use of antihypertensive medications. Therefore, our results suggest that IF/TA is a more relevant parameter than the other factors of increased mean values of both daytime and nighttime BP in this patient population.

In general, both the severity of IF/TA and frequency of GS increase in association with the progression of renal impairment. In fact, the IF/TA grade exhibited a trend toward an association with the GS grade in our current series (data not shown). Although our results showed a relationship between the grade of IF/TA and BP abnormalities on ABPM, no such relationships were observed between the grade of GS and the ABPM findings. This discrepancy may be explained by the possibility that globally sclerotic glomeruli merge and disappear over long-term periods; thus, the frequency of GS on biopsy specimens may be underestimated. ${ }^{14}$ Consistent with this idea are reports that the presence of IF/TA and not GS is the best histopathological parameter of the renal prognosis in patients with various progressive renal diseases. ${ }^{15-17}$ Importantly, the relationships between the grade of IF/ TA and the prevalence of daytime and nighttime hypertension on ABPM were independent of apparent renal dysfunction in the current study. These results suggest that the association between IF/TA and BP abnormalities does not simply reflect the loss of the entire renal function. Rather, our results suggest the possibility of intrarenal mechanisms controlling systemic BP at the tubulointerstitial level.

Johnson et al. ${ }^{18}$ proposed the hypothesis that injury to the peritubular capillaries is an important factor in inducing saltsensitive hypertension. They showed that tubulointerstitial injury induced by transient exposure to cyclosporine is associated with salt-sensitive hypertension in the absence of apparent renal dysfunction. ${ }^{19}$ Studies using experimental models of protein overload or cellophane wrapping (pressure injury) of the kidney resulted in saltsensitive hypertension, further supporting the hypothesis that the tubulointerstitium has an important role in the regulation of the BP in CKD patients. ${ }^{20,21}$ Recently, some possible mechanisms underlying the hypertension induced by the tubulointerstitium have been proposed. For example, Oppermann et al..$^{22}$ found that the angiotensin receptorassociated protein (Atrap), which decreases the effects of angiotensin II receptor type-1 by modulating its trafficking, is expressed in the kidneys, where it localizes in the proximal tubules. Atrap-knockout mice exhibit higher BP and plasma volume values than wild-type mice. ${ }^{22}$ Atrap-transgenic mice, which dominantly express Atrap in the renal tubules, do not display elevated $\mathrm{BP}$ values in response to angiotensin II infusion. ${ }^{23}$

It has been reported that the collecting duct produces large amounts of endothelin-1 that binds in an autocrine manner to the endothelin A and $\mathrm{B}$ receptors, thereby inhibiting sodium and water reabsorption. The absence of endothelin-1 or its receptors in the collecting duct was shown to be associated with marked salt-sensitive hypertension. ${ }^{24,25}$ The results of these experimental studies support the hypothesis that $\mathrm{BP}$ is, at least in part, mediated at the level of the renal tubulointerstitial region, which is consistent with our current findings that show a closer relationship between the grade of IF/TA and BP abnormalities than that observed for the other histopathological parameters.

The grade of $\mathrm{AO}$ exhibited significant associations with the daytime systolic, nighttime systolic and nighttime diastolic BP values according to the one-way analysis of variance. However, we did not find any significant trends regarding the grade of $\mathrm{AO}$ in the multivariate analysis or subgroup analyses of the patients without apparent renal impairment and those treated without antihypertensive drugs. However, the susceptibility to a sampling bias in the arterioarteriolar findings compared with those in the tubulointerstitial findings should be kept in mind. In addition, owing to the limited materials obtained by needle biopsies, we could not determine the intrarenal localization of the specimens and whether the IF/TA was derived from renal vascular lesions (ischemia) or glomerular lesions (glomerulosclerosis). Perera $^{26}$ suggested that the occurrence of arteriolar changes in the human kidney is a consequence of hypertensive vascular injury, as they found the severity of vascular lesions to be associated with the duration of hypertension. Castleman and Smithwick ${ }^{27}$ evaluated the renal biopsy specimens of patients with essential hypertension obtained during sympathectomy and found that $10-20 \%$ of the patients had only mild renal microvascular lesions. Together with these findings, our results suggest that AO may partially reflect renal vascular injury as a result of increased BP.

According to the results of the current multivariate analyses, male gender is significantly associated with both daytime and nighttime hypertension. In addition, further multivariate analyses including diabetes mellitus and current smoking status revealed that male gender was still a significant factor (data not shown). In general, a male gender may be associated with many confounding factors related to hypertension, such as hyperuricemia, smoking and sex hormone 
levels. ${ }^{28-31}$ It is also known that estradiol is protective against subclinical kidney damage and atherosclerosis. ${ }^{32,33}$ Although our study did not address all of the potential factors associated with gender, our results are fully consistent with the findings of previous large-scale studies, demonstrating that male gender is an independent risk factor for the progression of CKD. ${ }^{34-36}$

Our study showed weak but significant relationships between renal histopathological parameters and circadian BP abnormalities. Kimura and group ${ }^{37,38}$ proposed the hypothesis that nocturnal hypertension and resultant pressure natriuresis compensate for daytime sodium retention caused by impaired renal sodium excretion in CKD patients. All patients included in our study were Japanese. Approximately $40 \%$ of Japanese patients with essential hypertension have been estimated to have salt sensitivity; this rate is expected to be even higher among CKD patients. ${ }^{39,40}$ Because ABPM was performed under a sodiumrestricted diet during hospitalization in this study, there is a possibility that the trend toward circadian BP abnormalities was modified as a result of sodium restriction. Furthermore, it has been reported that the use of angiotensin type-1 receptor blockers modified the circadian BP measured by ABPM. ${ }^{41}$ Therefore, the use of antihypertensive drugs, including angiotensin type-1 receptor blockers, might have influenced the ambulatory BP in the current cohort.

The present study includes several limitations. First, the cause-effect relationships between the histopathological findings and BP abnormalities are unclear as this study was designed as a cross-sectional study. Second, our results may not be applied to all CKD patients because our analyses were limited to patients who underwent renal biopsies. Third, our results may have been modified by the use of bed rest and a sodium-restricted diet during the hospitalization period. Future studies performed at outpatient clinics will help to clarify the relationships between renal histopathological findings and actual ambulatory BP abnormalities in CKD patients.

In conclusion, among four candidate renal histopathological findings, including GS, IF/TA, AA and AO, only IF/TA showed significant associations with various abnormalities on $\mathrm{ABPM}$, including increased mean values of the daytime and nighttime BP, daytime and nighttime hypertension, and circadian BP abnormalities. Although the mechanisms are largely unknown at present, our current results indicate the possibility of tubulointerstitial injury as an origin of BP abnormalities in CKD patients.

\section{CONFLICT OF INTEREST}

The authors declare no conflict of interest.

\section{ACKNOWLEDGEMENTS}

Portions of this study were presented at the American Society of Nephrology Kidney Week, November 2012, San Diego, CA, USA and the World Congress of Nephrology, June 2013, Hong Kong.

1 Coresh J, Wei GL, McQuillan G, Brancati FL, Levey AS, Jones C, Klag MJ. Prevalence of high blood pressure and elevated serum creatinine level in the United States: findings from the third National Health and Nutrition Examination Survey (1988-1994). Arch Intern Med 2001; 161: 1207-1216.

2 Farmer CK, Goldsmith DJ, Cox J, Dallyn P, Kingswood JC, Sharpstone P. An investigation of the effect of advancing uraemia, renal replacement therapy and renal transplantation on blood pressure diurnal variability. Nephrol Dial Transplant 1997; 12: 2301-2307.

3 Davidson MB, Hix JK, Vidt DG, Brotman DJ. Association of impaired diurnal blood pressure variation with a subsequent decline in glomerular filtration rate. Arch Intern Med 2006; 166: 846-852.

4 Minutolo R, Agarwal R, Borrelli S, Chiodini P, Bellizzi V, Nappi F, Cianciaruso B, Zamboli P, Conte G, Gabbai FBDe Nicola L. Prognostic role of ambulatory blood pressure measurement in patients with nondialysis chronic kidney disease. Arch Intern Med 2011; 171: 1090-1098.

5 Agarwal R, Andersen MJ. Prognostic importance of ambulatory blood pressure recordings in patients with chronic kidney disease. Kidney Int 2006; 69: 1175-1180.

6 Kawai T, Ohishi M, Kamide K, Nakama C, Onishi M, Ito N, Takami Y, Takeya Y, Rakugi $H$. Differences between daytime and nighttime blood pressure variability regarding systemic atherosclerotic change and renal function. Hypertens Res 2013; 36: 232-239.

7 Kawai T, Kamide K, Onishi M, Yamamoto-Hanasaki H, Baba Y, Hongyo K, Shimaoka I, Tatara Y, Takeya Y, Ohishi M, Rakugi H. Usefulness of the resistive index in renal Doppler ultrasonography as an indicator of vascular damage in patients with risks of atherosclerosis. Nephrol Dial Transplant 2011; 26: 3256-3262.

8 Jonhson RJ, Schreiner G. Hypothesis: the role of acquired tubulointerstitial disease in the pathogenesis of salt-dependent hypertension. Kidney Int 1997; 52: 1169-1179.

9 Neumann J, Ligtenberg G, Klein II, Koomans HA, Blankestijn PJ. Sympathetic hyperactivity in chronic kidney disease: pathogenesis, clinical relevance, and treatment. Kidney Int 2004; 65: 1568-1576.

10 Matsuo S, Imai E, Horio M, Yasuda Y, Tomita K, Nitta K, Yamagata K, Tomino Y, Yokoyama $\mathrm{H}$, Hishida A. Collaborators developing the Japanese equation for estimated GFR Revised equations for estimated GFR from serum creatinine in Japan. Am J Kidney Dis 2009; 53: 982-992.

11 Ogihara T, Kikuchi K, Matsuoka H, Fujita T, Higaki J, Horiuchi M, Imai Y, Imaizumi T, Ito S, Iwao H, Kario K, Kawano Y, Kim-Mitsuyama Shimada K, Shimamoto K, Suzuki H, Takishita S, Tanahashi N, Tsuchihashi T, Uchiyama M, Ueda S, Ueshima H, Umemura $\mathrm{S}$, Ishimitsu T, Rakugi H. Japanese Society of Hypertension Committee The Japanese Society of Hypertension Guidelines for the Management of Hypertension (JSH 2009). Hypertens Res 2009 32: 3-107.

12 Kario K, Pickering TG, Matsuo T, Hoshide S, Schwartz JE, Shimada K. Stroke prognosis and abnormal nocturnal blood pressure falls in older hypertensives. Hypertension 2001; 38: 852-857.

13 Roberts IS, Cook HT, Troyanov S, Alpers CE, Amore A, Barratt J, Berthoux F, Bonsib S, Bruijn JA, Cattran DC, Coppo R, D'Agati V, D'Amico G, Emancipator S, Emma F, Feehally J, Ferrario F, Fervenza FC, Florquin S, Fogo A, Geddes CC, Groene HJ, Haas M, Herzenberg AM, Hill PA, Hogg RJ, Hsu SI, Jennette JC, Joh K, Julian BA, Kawamura T, Lai FM, Li LS, Li PK, Liu ZH, Mackinnon B, Mezzano S, Schena FP, Tomino Y, Walker PD, Wang H, Weening JJ, Yoshikawa N, Zhang H. Working Group of the International IgA Nephropathy Network and the Renal Pathology Society. The Oxford classification of IgA nephropathy: pathology definitions, correlations, and reproducibility. Kidney Int 2009; 76: 546-556.

14 Rule AD, Semret MH, Amer H, Cornell LD, Taler SJ, Lieske JC, Melton LJ 3rd, Stegall MD, Textor SC, Kremers WK, Lerman LO. Association of kidney function and metabolic risk factors with density of glomeruli on renal biopsy samples from living donors. Mayo Clin Proc 2011; 86: 282-290.

15 Cattran Daniel C, Coppo Rosanna, Terence Cook H, Feehally John, Roberts Ian SD., Troyanov Ste'phan, Alpers Charles E, Amore Alessandro, Barratt Jonathan, Berthoux Francois, Bonsib Stephen, Bruijn Jan A, D'Agati Vivette, D'Amico Giuseppe, Emancipator Steven, Emma Francesco, Ferrario Franco, Fervenza Fernando C, Florquin Sandrine, Fogo Agnes, Geddes Colin C, Groene Hermann-Josef, Haas Mark, Herzenberg Andrew M, Hill Prue A, Hogg Ronald J, Hsu Stephen I, Charles Jennette J, Joh Kensuke, Julian Bruce A., Kawamura Tetsuya, Lai Fernand M, Bon Leung Chi, Li Lei-Shi, Li Philip KT, Liu Zhi-Hong, Mackinnon Bruce, Mezzano Sergio, Paolo Schena F, Tomino Yasuhiko, Walker Patrick D, Wang Haiyan, Weening Jan J, Yoshikawa Nori, Zhang Hong. A Working Group of the International IgA Nephropathy Network and the Renal Pathology Society The Oxford classification of IgA nephropathy: rationale, clinicopathological correlations, and classification. Kidney Int 2009; 76: 534-545.

16 Okada T, Nagao T, Matsumoto H, Nagaoka Y, Wada T, Nakao T. Histological predictors for renal prognosis in diabetic nephropathy in diabetes mellitus type 2 patients with overt proteinuria. Nephrology 2012; 17: 68-75.

17 Wu Q, Jinde K, Nishina M, Tanabe R, Endoh M, Okada Y, Sakai H, Kurokawa K. Analysis of prognostic predictors in idiopathic membranous nephropathy. Am J Kidney Dis 2001; 37: 380-387.

18 Johnson RJ, Schreiner GF. Hypothesis: the role of acquired tubulointerstitial disease in the pathogenesis of salt-dependent hypertension. Kidney Int 1997; 52: 1169-1179.

19 Andoh TF, Johnson RJ, Lam T, Bennett WM. Subclinical renal injury induced by transient cyclosporine exposure is associated with salt-sensitive hypertension. Am J Transplant 2001; 1: 222-227.

20 Álvarez V, Quiroz Y, Nava M, Pons H, Rodríguez-lturbe B. Overload proteinuria is followed by salt-sensitive hypertension caused by renal infiltration of immune cells. Am J Physiol Renal Physiol 2002; 283: F1132-F1141.

21 Vanegas V, Ferrebuz A, Quiroz Y, Rodríguez-Iturbe B. Hypertension in Page (cellophane-wrapped) kidney is due to interstitial nephritis. Kidney Int 2005; 68: 1161-1170.

22 Oppermann M, Gess B, Schweda F, Castrop H. Atrap deficiency increases arterial blood pressure and plasma volume. J Am Soc Nephrol 2010; 21: 468-477.

23 Wakui H, Tamura K, Masuda S, Tsurumi-Ikeya Y, Fujita M, Maeda A, Ohsawa M, Azushima K, Uneda K, Matsuda M, Kitamura K, Uchida S, Toya Y, Kobori H, Nagahama K, Yamashita A, Umemura S. Enhanced angiotensin receptor-associated protein in renal tubule suppresses angiotensin-dependent hypertension. Hypertension 2013; 61: 1203-1210. 
24 Korbmacher C, Boulpaep EL, Giebisch G, Geibel J. Endothelin increases [Ca2+]i in M-1 mouse cortical collecting duct cells by a dual mechanism. Am J Physiol 1993; 265: C349-C357.

25 Kohan DE. Role of collecting duct endothelin in control of renal function and blood pressure. Am J Physiol Regul Integr Comp Physiol 2013; 305: R659-R668.

26 Perera GA. Hypertensive vascular disease; description and natural history. J Chronic Dis 1955; 1: 33-42.

27 Castleman B, Smithwick RH. The relation of vascular disease to the hypertensive state. Based on a study of renal biopsies from one hundred hypertensive patients. J Am Med Assoc 1943; 121: 1256-1261.

28 Cannon PJ, Stason WB, Demartini FE, Sommers SC, Laragh JH. Hyperuricemia in primary and renal hypertension. N Engl J Med 1966; 275: 457-464.

29 Schmidt MI, Watson RL, Duncan BB, Metcalf P, Brancati FL, Sharrett AR, Davis CE, Heiss G. Clustering of dyslipidemia, hyperuricemia, diabetes, and hypertension and its association with fasting insulin and central and overall obesity in a general population: Atherosclerosis Risk in Communities Study Investigators. Metabolism 1996; 45 699-706.

30 Hermida RC, Chayán L, Ayala DE, Mojón A, Fontao MJ, Fernández JR. Relationship between metabolic syndrome, circadian treatment time, and blood pressure nondipping profile in essential hypertension. Chronobiol Int 2011; 28: 509-519.

31 Malan NT, Hamer M, Lambert GW, Schutte AE, Huisman HW, Van Rooyen JM, Mels CM, Smith W, Fourie CM, Schutte R, Kruger R, Malan L. Sex hormones associatedwith subclinical kidney damage and atherosclerosis in South African men: the SABPA study. J Hypertens 2012; 30: 2387-2394.

32 Baylis C. Changes in renal hemodynamics and structure in the aging kidney; sexual dimorphism and the nitric oxide system. Exp Gerontol 2005; 40: 271-278.
33 Forte P, Kneale BJ, Milne E, Chowienczyk PJ, Johnston A, Benjamin N, Ritter JM Evidence for a difference in nitric oxide biosynthesis between healthy women and men. Hypertension 1998; 32: 730-734.

34 Neugarten J, Acharya A, Silbiger SR. Effect of gender on the progression of nondiabetic renal disease: a meta-analysis. J Am Soc Nephrol 2000; 11: 319-329.

35 Evans M, Fryzek JP, Elinder CG, Cohen SS, McLaughlin JK, Nyren O, Fored CM. The natural history of chronic renal failure: results from an unselected, population-based, inception cohort in Sweden. Am J Kidney Dis 2005; 46: 863-870.

36 Eriksen BO, Ingebretsen OC. The progression of chronic kidney disease: a 10-year population-based study of the effects of gender and age. Kidney Int 2006; 69: 375-382.

37 Kimura G. Sodium, kidney, and circadian rhythm of blood pressure. Clin Exp Nephrol 2001; 5: 13-18.

38 Fukuda M, Mizuno M, Yamanaka T, Motokawa M, Shirasawa Y, Nishio T, Miyagi S, Yoshida A, Kimura G. Patients with renal dysfunction require a longer duration until blood pressure dips during the night. Hypertension 2008; 52 $1155-1160$.

39 Weir MR, Fink JC. Salt intake and progression of chronic kidney disease: an overlooked modifiable exposure? A commentary. Am J Kidney Dis 2005; 45: 176-188.

40 Morimoto A, Uzu T, Fujii T, Nishimura M, Kuroda S, Nakamura S, Inenaga T, Kimura G. Sodium sensitivity and cardiovascular events in patients with essential hypertension. Lancet 1997; 350: 1734-1737.

41 Yanagi M, Tamura K, Fujikawa T, Wakui H, Kanaoka T, Ohsawa M, Azushima K, Maeda A, Kobori H, Umemura S. The angiotensin II type 1 receptor blocker olmesartan preferentially improves nocturnal hypertension and proteinuria in chronic kidney disease. Hypertens Res 2013; 36: 262-269.

Supplementary Information accompanies the paper on Hypertension Research website (http://www.nature.com/hr) 